\title{
Low arousal modulates visuospatial attention in three-dimensional virtual space
}

\author{
INES A. HEBER, ${ }^{1}$ JAKOB T. VALVODA, ${ }^{2}$ TORSTEN KUHLEN, ${ }^{2}$ AND BRUNO FIMM ${ }^{1}$ \\ ${ }^{1}$ Department of Neurology—Section Neuropsychology, University Hospital RWTH Aachen, Aachen, Germany \\ ${ }^{2}$ Virtual Reality Group, Center for Computing and Communication, RWTH Aachen University, Aachen, Germany
}

(Received March 27, 2007; Final Revision November 8, 2007; Accepted November 8, 2007)

\begin{abstract}
Clinical, experimental, and functional imaging studies suggest overlapping neuronal networks and functional interactions of alertness and visuospatial attention within the right hemisphere of the brain. To examine the interaction of arousal and visuospatial attention in peripersonal and extrapersonal virtual space, we tested 20 healthy male adults during $24 \mathrm{hr}$ of sleep deprivation at four points during the night (9 p.m., 1 a.m., 5 a.m., and 9 a.m.). The main finding concerning covert orienting in a virtual environment is a highly significant slowing of reorientation toward the left visual hemifield in extrapersonal space due to decreased arousal. The results provide additional evidence for the proposed anatomical and functional overlap of the two attentional systems and indicate a modulation of visuospatial attention by the level of arousal in extrapersonal space. (JINS, 2008, 14, 309-317.)
\end{abstract}

Keywords: Attentional asymmetry, Alertness, Orienting of attention, Virtual reality, Sleep deprivation, Depth perception

\section{INTRODUCTION}

Clinical studies indicate that symptoms of neglect can be associated with lesions of the attentional alertness network. This finding is supposed to be caused by its substantial overlap with the posterior orienting network within the right cerebral hemisphere (Bartolomeo, 2000; De Renzi et al., 1989; Gitelman et al., 2002; Karnath et al., 1998; Vallar, 2001). While positron emission tomography (PET) and functional magnetic resonance imaging (fMRI) studies suggest a righthemisphere frontal and inferior parietal network subserving alertness (Fernandez-Duque \& Posner, 2001; Sturm et al., 1999; Sturm \& Willmes, 2001), the orienting network is associated with right frontal and superior, as well as inferior parietal cortical areas (Gitelman et al., 1999; Posner et al., 1984; Vallar et al., 2003). Additionally, it is subserved by subcortical structures such as the superior colliculus of the midbrain and the pulvinar and reticular nucleus of the thalamus (Posner \& Petersen, 1990).

Correspondence and reprint requests to: Ines Ann Heber, RWTH Aachen University, Department of Neurology, Section Neuropsychology, Pauwelsstraße 30, D-52074 Aachen. E-mail: heber@ neuropsych.rwth-aachen.de
Indications of a functional coupling of the alerting and the orientation network arise from clinical findings as well as from experimental and rehabilitation studies. Robertson et al. (1998) were able to show a positive influence of alertness training on neglect symptoms. They proved that unlateralized warning tones, used to phasically increase alertness in neglect patients, improved their symptoms by diminishing their rightward bias of visuospatial attention. Thimm et al. (2006) demonstrated how computerized alertness training can improve visuospatial performance of neglect patients and that this is linked to reactivation in right hemisphere brain areas (frontal cortex, anterior cingulate cortex, precuneus, and angular gyrus) associated with alerting and visuospatial attention. Bellgrove et al. (2004) demonstrated how individual differences in alertness capacity can modulate pseudoneglect (the small leftward attentional bias in healthy subjects), whereas Callejas et al. (2004) identified an accelerating influence of alerting on orienting. In a recent study (Fimm et al., 2006), we provoked attentional asymmetries in healthy subjects by short-term sleep deprivation ( $28 \mathrm{hr}$ ) leading to a substantial reduction of arousal associated with a significant slowing of responses to stimuli presented to the left visual hemifield as well as a facilitation of covert reorienting of attention toward the right visual hemifield. 
While a functional link of alertness and visuospatial attention has been demonstrated for patients and healthy subjects, all of the cited studies only investigated spatial attention in near (peripersonal) space. Traditionally, peripersonal space is defined as the space within arm's reach and is closely linked with planning and execution of manual movements and localization of objects (Goodale \& Milner, 1992; Ladavas \& Farne, 2004), whereas far (extrapersonal) space is defined as the space outside arm's reach and preoccupied with object identification, although these assignments can be partially modified (Berti et al., 2001; Longo \& Lourenco, 2006). The dorsal visual stream primarily contributes to visual perception and attention to peripersonal space as well as to manual action in space, whereas the ventral visual stream is associated with attention to events in far extrapersonal space (Previc, 1998; Weiss et al., 2000). Some neglect patients show dissociations of impairment between peripersonal and extrapersonal visual space, with selective impairment within one and normal performance within the other spatial domain. These findings reflect the different contributions of the dorsal and ventral streams of visual processing to visuospatial attention. Brain (1941) was the first to report a case of neglect restricted to peripersonal space. Other studies assessing radial line bisection reported patients with "far" (Shelton et al., 1990; Vuilleumier et al., 1998) or "near" (Halligan \& Marshall, 1991; Mennemeier et al., 1992) neglect symptoms, depending on lesion site and linking far-upper attentional neglect to lesions of the inferior temporal cortex and near-lower neglect symptoms to posterior parietal lesions. Butler et al. (2004) detected lateral gradients of increasing target detection from left to right in both peripersonal and extrapersonal space and identified relations between peripersonal neglect and dorsal stream lesions and extrapersonal neglect and ventral stream damage, respectively. In a combined line bisection and PET study, Weiss et al. (2000), identified neural activations in the left dorsal occipital cortex, left intraparietal cortex, left ventral premotor cortex, and left thalamus upon performance in near space and bilateral ventral occipital cortex and right medial temporal cortex activations upon performance in far space. Transcranial magnetic stimulation of the right posterior parietal cortex and the right ventral occipital lobe provoked significant rightward shifts of the perceived midpoint in a line bisection task in near and far space, respectively (Bjoertomt et al., 2002), thus underlining the near-dorsal and far-ventral segregation of visual processing. Maringelli et al. (2001) examined the distribution of visuospatial attention in a Virtual Reality (VR) setup using a head-mounted display and showed how a virtual representation of one's own body affected the distribution of attention by provoking a bias of visuospatial attention toward near body-centered space in presence and an attentional bias toward far space in absence of the virtual body. Losier and Klein (2004) also used a VR setup to investigate covert attention in peripersonal and extrapersonal space. They report an advantage for lower field targets in peripersonal space consistent with the proposed bias by Previc
(1998). Both VR studies did not report any horizontal attentional biases.

Despite extensive research in the field of visuospatial attention, attentional visual field asymmetries in the two different spatial zones have never been examined in relation to arousal, although findings might be highly interesting in regard to the underlying spatial systems, their role in neglect and their relevancy for therapeutic approaches of different neglect characteristics. In this study, we systematically manipulated the level of arousal by sleep deprivation and investigated its effect on visuospatial attention in virtual space in healthy subjects.

Given that the networks of alertness and attention share common anatomical structures within the inferior parietal cortex and considering previous findings within our group (Fimm et al., 2006), as well as the alertness training studies of Robertson et al. (1998) and Thimm et al. (2006), we expect an influence of decreasing alertness on visuospatial attention in both peri- and extrapersonal space, albeit to a different extend. The reported clinical and experimental dissociations of neglect symptoms suggest that the intensity of potential attentional asymmetries between the left and the right visual field might vary subject to the depth of stimuli presentation. Additionally, there is strong evidence that the posterior parietal cortex is more densely innervated by activating afferences of the locus coeruleus (LC) than the temporal lobe (Morrison \& Foote, 1986) and sleep deprivation is assumed to lead to widespread decreases in global and regional cerebral metabolic rate of glucose (CMRglu) in the posterior parietal lobe (Thomas et al., 2000). These significant CMRglu reductions in sleep-deprived subjects were specifically found in areas involved in alertness, attention and other cognitive functions, namely in the thalamus and the prefrontal and the posterior parietal cortices. Accordingly, we expect the dorsal pathway to be more affected by low arousal than the ventral pathway, thus resulting in a more pronounced attentional asymmetry effect within the applied covert attention paradigm in peripersonal space. Because the posterior parietal cortex is crucial in redirecting attention to previously unattended locations (Petersen et al., 1989; Posner et al., 1984; Thiel et al., 2004), we also expect a stronger asymmetry effect for invalidly cued targets.

\section{METHOD}

\section{Subjects}

Twenty men with an age range of 21 to 31 years (mean = 26.2 years) took part in the study. All subjects were nonsmokers, right-handed [according to a German version of the Edinburgh Handedness Inventory (Oldfield, 1971)], without any former history of neurological or psychiatric disease, and all reported normal depth perception and normal or corrected-to-normal visual acuity. All persons gave their informed consent to the participation in the study, which was approved by the local research ethics committee. 


\section{Procedure}

Participants were instructed to sleep a minimum of 6 and a maximum of $8 \mathrm{hr}$ in the night before the study, to get up no later than 9 a.m. as well as to avoid any stimulating substances and medication on the day of the study. The participants arrived at the lab at 4:30 p.m. At the beginning of the study protocol, subjects were tested for their ability to distinguish between different levels of virtual depth and peripersonal versus extrapersonal space with a short VR paradigm (Armbrüster et al., 2005), which required distance estimations of ten spheres of equal retinal sizes in distances between 30 and $330 \mathrm{~cm}$. All participants succeeded in sequencing the stimuli according to their distance and judging the spatial realms. Additionally, at 5 p.m. as well as at 7 p.m., they were tested for their ability to covertly orient attention with the subtest "Covert shift of attention" of the Test for Attentional Performance (TAP; Zimmermann \& Fimm, 2002). Only subjects with good abilities in depth perception, eye fixation (which was visually controlled by the experimenter), and displaying a sufficient validity effect ( $>20 \mathrm{~ms}$ ) in the covert orienting task were included in the study and completed the virtual reality paradigms repeatedly throughout the night. Two subjects were excluded because of deficits in covert orienting and the investigation was aborted after the 5 p.m. session.

The main experimental sessions were carried out at 9 p.m., 1 a.m., 5 a.m., and 9 a.m. During the breaks, participants were allowed to drink, eat, read, surf the Internet, and listen to music. They were not allowed to take any naps or consume any stimulating beverages or medications and were constantly monitored by the investigator.

\section{Apparatus and Software}

The virtual setup was displayed on a BARCO Baron ${ }^{\mathrm{TM}}$ rear projection desk. An active stereo system was used to enable immersive stereoscopic visualization. The participants wore shutter glasses, which enable three-dimensional (3D) perception of projected stimuli. The subject's head was fixated by a chin rest to reduce head movements and changes of perspective. Additionally, head movements were tracked with an electromagnetic head-tracker (Flock of Birds ${ }^{\mathrm{TM}}$ ) to monitor the subjects, but also to enhance the subject's depth perception by minimal motion parallax.

Because timing precision in common computer operating systems can be critical for psychological reaction time experiments (Myors, 1999), a special reaction-time hardware was developed (Valvoda et al., 2004; Wolter et al., 2007). This system provides data with potential sampling and delay errors below $0.01 \mathrm{~ms}$, and thus enables the realization of real-time reaction-time experiments in platformindependent virtual environments.

To generate the Virtual Reality paradigm, the ReactorMan software - a part of the NeuroMan system (Valvoda et al., 2003)—was used. ReactorMan enables the definition of setups for VR-based experiments, provides information about the runtime behavior of the software and the participants' reactions to events and interaction devices and together with the reaction-time hardware features the possibility to $\log$ the overall chronological behavior with specific timing characteristics (Valvoda et al., 2004).

\section{Task}

The task consisted of a covert attention paradigm following the covert attention tasks of Posner (Posner, 1980; Posner et al., 1984) in a 3D Virtual Reality environment. Four white-shaded balls were presented in a unichrome blue virtual space at two different planes of depth: two in peripersonal $(50 \mathrm{~cm})$ and two in extrapersonal space $(240 \mathrm{~cm})$, one per plane of depth in left and one in right visual hemispace, respectively $\left(23^{\circ}\right.$ and $17^{\circ}$ visual angle from the fixation point in extrapersonal and peripersonal space). A small green star in the middle of the resulting rectangle served as a fixation point (see Figure 1).

While the participants were instructed to keep their eyes fixed on the green star, one of the four balls changed its color to yellow and slightly decreased in its size for $150 \mathrm{~ms}$ to induce a covert shift of attention toward the cued location. After a pseudorandomized cue-target interval of 250 , 300,350 , or $400 \mathrm{~ms}$, one of the balls changed to red, indicating the target stimulus. Subjects were instructed to press a button with their right index finger as quickly as possible upon detecting the target. To ensure predictability of the target location, it appeared at the cued location with a probability of $70 \%$ and accordingly at one of the uncued locations with a probability of $30 \%$. Thus, invalidly cued targets required a covert shift of attention either horizontally (e.g., peripersonal right to peripersonal left), diagonally (e.g., peripersonal right to extrapersonal left) or radially (e.g., extrapersonal left to peripersonal left) across the 3D visual space. With three pseudorandomized intertrial intervals $(1500,2000,2500 \mathrm{~ms})$, the whole session consisted

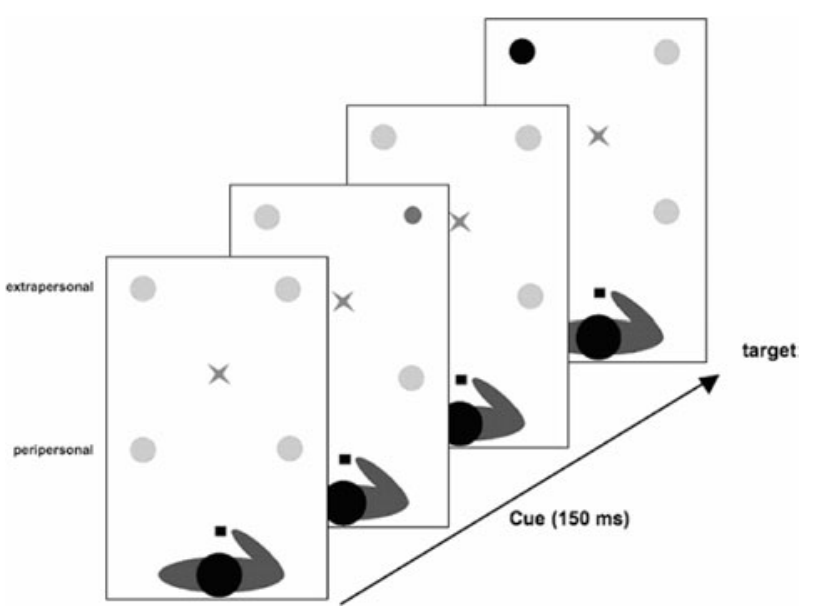

Fig. 1. Diagram of an invalid trial in the covert attention paradigm. 
of 144 invalid trials (12 per condition) and 336 valid trials, which summed up to 480 trials and lasted for approximately $25 \mathrm{~min}$. The task was presented every $4 \mathrm{hr}$ during the night (9 p.m., 1 a.m., 5 a.m., and 9 a.m.), resulting in a total duration of sleep deprivation of approximately $24 \mathrm{hr}$.

Additionally, participants' body temperature was measured at the beginning of each session with a digital in-ear thermometer (Braun IRT 3520 ThermoScan, Type 6012) as an indicator of physiological arousal (Van Someren, 2000). They were also asked to rate their mood status on a standardized questionnaire, the Befindlichkeitsskala BfS (von Zerssen, 1976), as well as their fatigue (as a measure of subjective arousal) on a combined 5/50 rating scale (Heller, 1985), which asked the subjects to first rate their fatigue on a verbal scale and then to refine the intensity of the fatigue within the category (wide awake $=0-10$, awake $=10-20$, neither awake nor tired $=20-30$, tired $=30-40$, extremely tired $=40-50$ ).

\section{Statistical Analysis}

To compare the participants' performance in the sessions with the highest and the lowest levels of arousal, depending on the amount of sleep deprivation, the data were analyzed with the SPSS package (version 14.0) computing Pearson correlations and a repeated measures analyses of variance for the reaction times with the factors validity (validly cued targets vs. horizontally, radially, and diagonally invalidly cued targets), side (target position in the left $v s$. right visual hemifield), depth (peripersonal $v s$. extrapersonal space), and time (of experimental session; 9 p.m., 1 a.m., 5 a.m., 9 a.m.). Significance levels were adjusted with the GreenhouseGeisser correction when appropriate. Stimulus-onset asynchrony was not included as a factor due to undersized amounts of data within the resulting cells. However, preliminary separate analyses did not detect any interactions with one of the other factors. Over all, reaction times faster than $100 \mathrm{~ms}$ (anticipative reactions) and slower than $1000 \mathrm{~ms}$ (distinct delayed reactions) were excluded from the analysis, representing $9.5 \%$ of all trials. Median reaction times were log-transformed to correct for skewness of distribution before including them in the repeated measures analysis of variance.

\section{RESULTS}

\section{Descriptive Measures of Body Temperature, Fatigue, and Mood}

The repeated measures analyses of variance for body temperature $\left(F_{1,19}=24.447 ; p<.001\right)$, fatigue $\left(F_{1,19}=46.859\right.$; $p<.001)$, and $\operatorname{mood}\left(F_{1,19}=26.559 ; p<.001\right)$ all reveal highly significant main effects of time. Mean average body temperature was maximal at the 9 p.m. session $\left(36.65^{\circ} \mathrm{C}\right)$, reached the minimum at 5:00 a.m. $\left(36.05^{\circ} \mathrm{C}\right)$ and slightly increased again at 9 a.m. $\left(36 \cdot 18^{\circ} \mathrm{C} ; 9\right.$ p.m. vs. 1 a.m.: $F_{1,19}=$ 30.425; $p<.001 ; 1$ a.m. vs. 5 a.m.: $F_{1,19}=42.506 ; p<$ $.001 ; 5$ a.m. vs. 9 a.m.: $F_{1,19}=4.957 ; p<.05$; reverse Helmert contrasts). The mean average of fatigue was lowest at 9 p.m. and highest during the last session at 9 a.m. The amount of fatigue increased highly significantly from one session to the next one, except for the last session $(9$ p.m. vs. 1 a.m.: $F_{1,19}=100.881 ; p<.001 ; 1$ a.m. vs. 5 a.m.: $F_{1,19}=64.266 ; p<.001 ; 5$ a.m. vs. 9 a.m.: $F_{1,19}=29.679$; $p<.001$; reverse Helmert contrasts). A similar profile could be observed for the mood values resulting in lowest ratings (good mood) at 9 p.m. and highest ratings (bad mood) at 9 a.m., respectively (9 p.m. vs. 1 a.m.: $F_{1,19}=23.688 ; p<$ $.001 ; 1$ a.m. vs. 5 a.m.: $F_{1,19}=34.170 ; p<.001 ; 5$ a.m. $v s$. 9 a.m.: $F_{1,19}=21.904 ; p<.001$; reverse Helmert contrasts). Self-reports of fatigue and mood correlate (Pearson product-moment correlation) significantly at 1 a.m. $(r=$ $.488 ; p<.05$ two-sided), 5 a.m. $(r=.451 ; p<.05$ twosided), and 9 a.m. ( $r=.624 ; p<.01$ two-sided $)$, indicating that sleep deprivation is associated with the emotional state of the participants. Both increases of subjective measures also correspond well with the decrease of body temperature, except for its slight increase at 9 a.m. (see Figure 2), although it was still significantly $\left(F_{1,19}=78.235 ; p<.001\right)$ below the baseline at this point.

\section{Behavioral Measures of Covert Attention}

A $4 \times 2 \times 2 \times 4$ (factors: validity, side, depth, and time) repeated measures analysis of variance of reaction times resulted in highly significant effects of VALIDITY $\left(F_{3,57}=\right.$ $76.809 ; p<.001)$, DEPTH $\left(F_{1,19}=16.548 ; p<.001\right)$, and
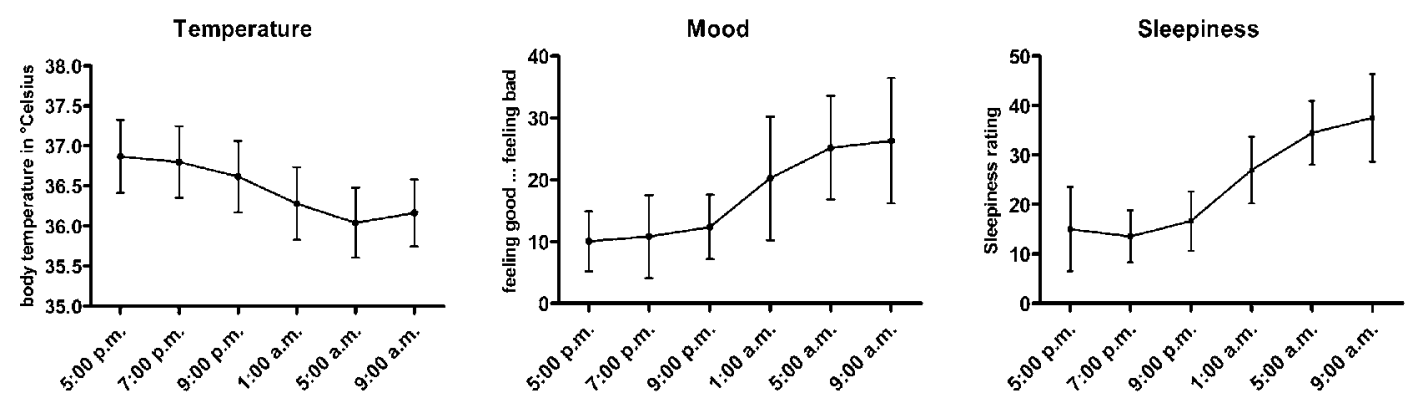

Fig. 2. Measures of temperature, self-reported mood, and fatigue. 
TIME $\left(F_{3,57}=10.431 ; p<.001\right)$. Highly significant simple contrasts of VALIDITY can be found for all three types of invalid cues, showing a most pronounced validity effect for diagonal reorientation $\left(F_{1,19}=211.427 ; p<.001\right)$ followed by horizontal reorientation $\left(F_{1,19}=158.416\right.$; $p<$ $.001)$ and radial reorientation $\left(F_{1,19}=62.791 ; p<.001\right.$; see Figure 3 ). The effect indicates that the VR paradigm was highly capable of initiating covert shifts of attention in virtual space.

The subsequent statistical analyses are based on the 9 p.m. and 9 a.m. sessions, which are the sessions with the shortest and longest amount of sleep deprivation, highest and lowest self-reported levels of arousal, body temperature significantly below baseline, and slowest overall reaction times (see Figure 3), revealed by a simple repeated measures analysis of variance of reaction times with the factor time, resulting in a highly significant effect of sleep deprivation on overall reaction times $\left(F_{3,57}=11.119 ; p<\right.$ $.001)$, as well as significant increases of reaction time from one session to the other (9 p.m. vs. 1 a.m.: $F_{1,19}=4.812$; $p<.05$; 1 a.m. vs. 5 a.m.: $F_{1,19}=18.799 ; p<.001 ; 5$ a.m. vs. 9 a.m.: $F_{1,19}=8.895 ; p<.01$; reverse Helmert contrasts).

\section{Comparison of High (9 p.m.) and Low (9 a.m.) Levels of Arousal}

The $4 \times 2 \times 2 \times 2$ (factors: validity, side, depth, and time) repeated measures analysis of variance of reaction times reveals significant effects of VALIDITY $\left(F_{3,57}=51.203\right.$; $p<.001)$, DEPTH $\left(F_{1,19}=7.535 ; p<.01\right)$, and TIME $\left(F_{1,19}=15.073 ; p<.001\right)$. The highly significant main effect of VALIDITY indicates that covert shifts of attention were initiated by the paradigm and the main effect of TIME demonstrates a general increase of reaction times over the course of the night (see Figure 3).

Additionally, two-way VALIDITY-by-SIDE $\left(F_{3,57}=\right.$ $4.745 ; p<.01)$, VALIDITY-by-DEPTH $\left(F_{3,57}=3.497\right.$; $p<.05)$, and SIDE-by-TIME $\left(F_{1,19}=4.661 ; p<.05\right)$, as well as three-way interactions of VALIDITY-by-DEPTH-

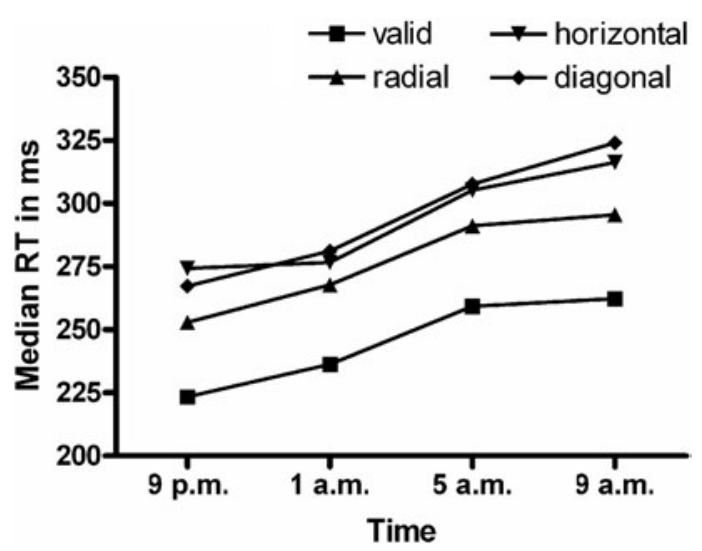

Fig. 3. Median reaction times (RT) of valid, radially, horizontally, and diagonally invalidly cued trials.
by-TIME $\left(F_{3,57}=3.099 ; p<.05\right)$, and VALIDITY-by-SIDEby-TIME $\left(F_{3,57}=4.318 ; p<.01\right)$ were significant. Last but not least, the four-way interaction of VALIDITY-by-SIDEby-DEPTH-by-TIME $\left(F_{3,57}=3.364 ; p<.05\right)$ is significant as well and the subsequent analysis and interpretations will concentrate on it.

To further explore this significant four-way interaction of VALIDITY-by-SIDE-by-DEPTH-by-TIME $\left(F_{3,57}=\right.$ $3.364 ; p<.05)$ separate analyses for peripersonal and extrapersonal targets were computed. Thus, the analysis was first split into two multivariate analyses of variance (MANOVAs) with three main factors (see Kirk, 1994). In case of a significant Validity-by-Side-by-Time interaction, the analysis was then further split into 2 MANOVAS based on 9 p.m. and 9 a.m., respectively, with two main factors (Validity, Side) each.

Whereas the VALIDITY-by-SIDE-by-TIME interaction in peripersonal space was not significant, the respective interaction in extrapersonal space proved to be significant $\left(F_{3,57}=4.858 ; p<.05\right)$. Subsequent separate analysis for extrapersonal targets at 9 p.m. and 9 a.m. yielded a significant VALIDITY-by-SIDE interaction at 9 a.m. $\left(F_{3,57}=\right.$ $6.765 ; p<.01)$. According to simple a priori contrasts, this was based on horizontal conditions (horizontal vs. valid: $F_{1,19}=9.953 ; p<.01$ ), whereas radial and diagonal conditions did not contribute significantly to this effect. Thus, the VALIDITY-by-SIDE-by-DEPTH-by-TIME interaction is mainly caused by a strong reaction time asymmetry to the disadvantage of left-sided targets at 9 a.m. when attention has to be shifted horizontally from right extrapersonal to left extrapersonal space (see Figure 4 and Table 1).

\section{DISCUSSION}

In our study, the maximally reduced level of arousal, induced by $24 \mathrm{hr}$ of sleep deprivation, resulted in slowed reorientation of attention toward horizontal invalidly cued targets within the left extrapersonal visual hemifield. The results provide further evidence for the postulated influential role of the alertness network on the network of visuospatial attention (Bellgrove et al., 2004; Fimm et al., 2006; Manly et al., 2005; Robertson et al., 1998) in a 3D virtual reality setting. Thus, we were able to replicate previous findings of a link between low arousal and attentional asymmetries (Fimm et al., 2006). Furthermore, our data suggest an exclusive influence of arousal on reorientation of attention (invalid trials), which includes the mechanisms of disengaging, moving, and engaging attention at the new location (Posner et al., 1984; Posner \& Petersen, 1990). Although reaction times of the initial orientation to validly cued targets were significantly increased at 9 a.m., there was no significant asymmetry between leftand right-sided correctly cued targets and attentional orienting was not affected by different levels of arousal, which is consistent with our preceding hypothesis. This finding is also in line with event-related fMRI studies (Corbetta et al., 2000; Thiel et al., 2004), which identified different neuronal networks subserving attentional aspects of alerting, orienting, 

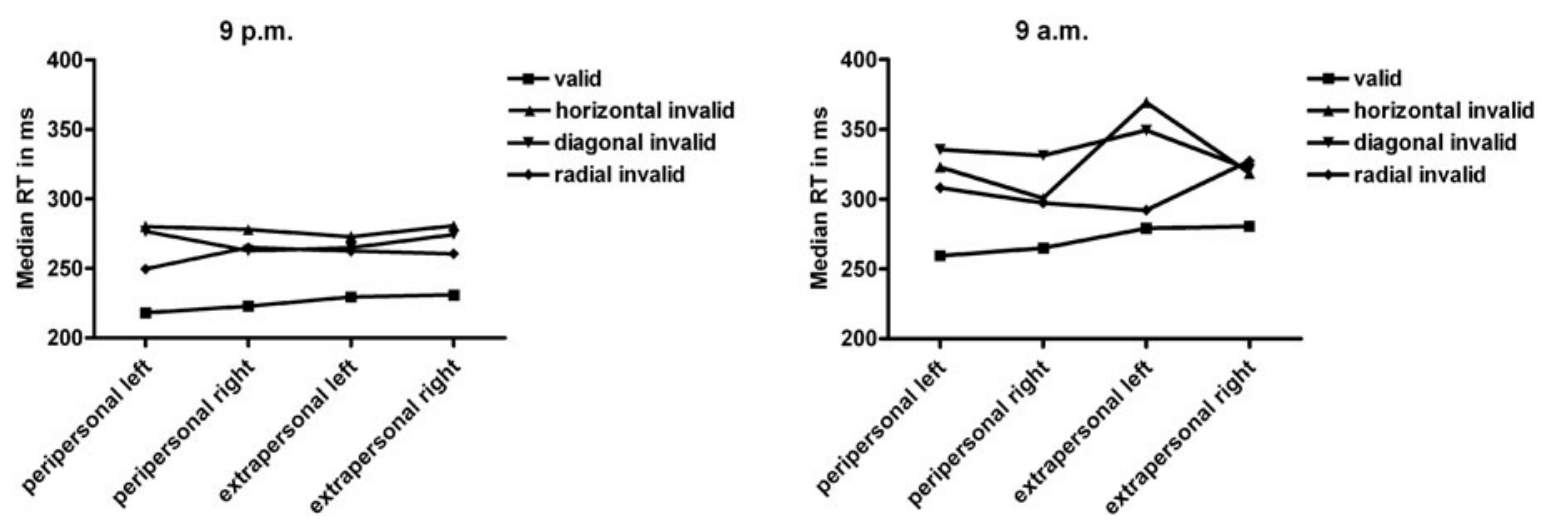

Fig. 4. Median reaction times (RT) of the significant validity-by-side-by-depth-by-time interaction.

Table 1. Mean reaction times (in ms) of all conditions $(n=20)$

\begin{tabular}{|c|c|c|c|c|c|}
\hline & & \multicolumn{2}{|c|}{ Peripersonal } & \multicolumn{2}{|c|}{ Extrapersonal } \\
\hline & & Left & Right & Left & Right \\
\hline Valid & 9 p.m. & 218 & 223 & 230 & 231 \\
\hline Invalid-radial & & 250 & 265 & 263 & 261 \\
\hline Invalid-horizontal & & 280 & 278 & 273 & 281 \\
\hline Invalid-diagonal & & 277 & 263 & 265 & 274 \\
\hline Valid & 9 a.m. & 260 & 265 & 279 & 281 \\
\hline Invalid-radial & & 308 & 297 & 292 & 327 \\
\hline Invalid-horizontal & & 323 & 301 & 369 & 319 \\
\hline Invalid-diagonal & & 336 & 331 & 349 & 321 \\
\hline
\end{tabular}

and reorienting and especially showed a clear dissociation of orienting and reorienting of visuospatial attention. Additionally, the right temporoparietal cortical junction (TPJ) was repeatedly identified as a key structure for reorientation of attention to previously unattended locations in clinical studies, demonstrating that lesions in this brain area are often associated with neglect symptoms (Friedrich et al., 1998; Thiebaut de Schotten et al., 2005; and in imaging studies: Corbetta \& Shulman, 2002; Corbetta et al., 2005; overview in: Danckert $\&$ Ferber, 2006). The right TPJ seems to play a crucial role in the observations made by this study. While the posterior parietal lobe generally receives strong noradrenergic (NA) projections, especially the right TPJ is densely innervated by ascending NA neurons of the $\mathrm{LC}$, which is the main source of NA innervation of the cortex (Beane \& Marrocco, 2004; Marrocco et al., 1994; Morrison \& Foote, 1986). Furthermore, the frontoparietal alertness network in the right hemisphere receives strong and widespread NA projections from the LC (Posner \& Petersen, 1990), emphasizing the role of NA projections in both orienting and alertness functions. AstonJones and Cohen (2005) suggest that one role of the LC system is to substantially contribute to the optimization of behavioral performance with phasic LC activity being closely tied to tonic LC activity (Aston-Jones et al., 1999). Coull et al. (2001) provided further evidence for the predominant role of the right hemisphere in arousal and attentional orienting using a covert orienting task. They showed how the adrenergic agonist clonidine modulated behavioral as well as neuroanatomical correlates of human attentional orienting and alerting by attenuating the cost of invalidly cued targets in the left but not in the right visual field. This was associated with a decrease of right superior parietal activation during spatial orienting. Subsumed, we conclude that the LC and the TPJ might provide the anatomical link for the mediating influence of the alertness network on the network of visuospatial attention.

In our study, we hypothesized a differential influence of low arousal on orienting of attention within the two spatial domains with a stronger asymmetry effect in peripersonal space. Contrary to our original hypothesis, it turned out to be the extrapersonal space being significantly affected by low arousal with respect to reorientation to horizontal invalidly cued targets.

This observation together with present clinical evidence (Halligan \& Marshall, 1991; Mennemeier et al., 1992; Shelton et al., 1990; Vuilleumier et al., 1998) points to a need for further, more detailed research. For instance, it would be highly interesting to distinguish between multiple (more than two as in our study) layers of depth, as well as different heights in the visual field representing different aspects of visuospatial perception and action. Previc (1998) gives an overview on different models of parcelling 3D space, 
and proposes four major zones of action: peripersonal, focal extrapersonal, action extrapersonal, and ambient extrapersonal space. According to this model, the extrapersonal distance of 2.40 meters in our study corresponds with 'action extrapersonal' space. Although no direct manual action can be carried out in this space, objects in this distance can quickly be accessed by moving toward them. Therefore, "action extrapersonal" space might be somewhat differently processed in the brain from "ambient extrapersonal" space, and visuospatial attention within this domain could be differentially modulated by low arousal, respectively. Another issue might also complicate the interpretation of the obtained results. Although we found main effects and interactions of depth (peripersonal vs. extrapersonal), the fixation point was located $145 \mathrm{~cm}$ from the subjects, beyond peripersonal in extrapersonal space. This might have confounded respective dorsal and ventral stream activations (Quinlan \& Culham, 2007) and possibly prevented a significant effect on reorientation in peripersonal space. Subsequent studies of visuospatial attention in virtual space (Heber et al., 2007) will have to account for this and keep fixation within the same depth plane of cues and targets.

In conclusion, our results provide a further step in understanding the relationship between orienting and alerting and emphasize the assumption of right hemisphere dominance and the anatomical and functional overlap of the two attentional networks of alerting and orienting. We were able to replicate previous findings of the direct influence of a low arousal level induced by sleep deprivation on visuospatial attention (Fimm et al., 2006), and we were able to show that the smaller but neglect-resembling effect appears in extrapersonal space. These findings are relevant for the rehabilitation of neglect or attentional asymmetries in 3D space (Butler et al., 2004; Halligan \& Marshall, 1991; Mennemeier et al., 1992; Robertson et al., 1998; Shelton et al., 1990; Thimm et al., 2006; Vuilleumier et al., 1998). Furthermore, the functional interdependence of the two attentional networks might be relevant to Attention Deficit Disorders as well, because various studies (Dobler et al., 2003, 2005; Manly et al., 2005; Nigg et al., 1997; Sheppard et al., 1999; Voeller \& Heilman, 1988) describe neglect symptoms or visual space biases in children with Attention Deficit Hyperactivity Disorder; potential therapeutic approaches might account for the functional overlap of alertness and visuospatial attention.

\section{ACKNOWLEDGMENTS}

This research was supported by a grant from the Interdisciplinary Center for Clinical Research "BIOMAT" within the faculty of Medicine at the RWTH Aachen University (TV N43). We would also like to thank the anonymous reviewers for valuable comments on previous versions of our manuscript.

\section{REFERENCES}

Armbrüster, C., Heber, I., Valvoda, J.T., Kuhlen, T., Fimm, B., \& Spijkers, W. (2005). Distance estimations in a VR application:
Interindividual differences and interindividual stabilities from a psychological point of view. Proceedings of the 11th international Conference on Human-Computer Interaction, Las Vegas, USA, 2005. CD-ROM.

Aston-Jones, G. \& Cohen, J.D. (2005). An integrative theory of locus coeruleus-norepinephrine function: Adaptive gain and optimal performance. Annual Review of Neuroscience, 28, 403-450.

Aston-Jones, G., Rajkowski, J., \& Cohen, J. (1999). Role of locus coeruleus in attention and behavioral flexibility. Biological Psychiatry, 46, 1309-1320.

Bartolomeo, P. (2000). Inhibitory processes and spatial bias after right hemisphere damage. Neuropsychological Rehabilitation, 10, 511-526.

Beane, M. \& Marrocco, R. (2004). Cholinergic and noradrenergic inputs to the posterior parietal cortex modulate the components of exogenous attention. In M.I. Posner (Ed.), Cognitive Neuroscience ofAttention (pp.313-325). New York: The Guilford Press.

Bellgrove, M.A., Dockree, P.M., Aimola, L., \& Robertson, I.H. (2004). Attenuation of spatial attentional asymmetries with poor sustained attention. Neuroreport, 15, 1065-1069.

Berti, A., Smania, N., \& Allport, A. (2001). Coding of far and near space in neglect patients. Neuroimage, 14, 98-102.

Bjoertomt, O., Cowey, A., \& Walsh, V. (2002). Spatial neglect in near and far space investigated by repetitive transcranial magnetic stimulation. Brain, 125, 2012-2022.

Brain, W.R. (1941). Visual disorientation with special reference to lesions of the right hemisphere. Brain, 64, 224-272.

Butler, B.C., Eskes, G.A., \& Vandorpe, R.A. (2004). Gradients of detection in neglect: Comparison of peripersonal and extrapersonal space. Neuropsychologia, 42, 346-358.

Callejas, A., Lupianez, J., \& Tudela, P. (2004). The three attentional networks: On their independence and interactions. Brain and Cognition, 54, 225-227.

Corbetta, M., Kincade, M.J., Lewis, C., Snyder, A.Z., \& Sapir, A. (2005). Neural basis and recovery of spatial attention deficits in spatial neglect. Nature Neuroscience, 8, 1603-1610.

Corbetta, M., Kincade, J.M., Ollinger, J.M., McAvoy, M.P., \& Shulman, G.L. (2000). Voluntary orienting is dissociated from target detection in human posterior parietal cortex. Nature Neuroscience, 3, 292-297.

Corbetta, M. \& Shulman, G.L. (2002). Control of goal-directed and stimulus-driven attention in the brain. Nature Reviews. Neuroscience, 3, 201-215.

Coull, J.T., Nobre, A.C., \& Frith, C.D. (2001). The noradrenergic $\alpha 2$ agonist clonidine modulates behavioural and neuroanatomical correlates of human attentional orienting and alerting. Cerebral Cortex, 11, 73-84.

Danckert, J. \& Ferber, S. (2006). Revisiting unilateral neglect. Neuropsychologia, 44, 987-1006.

De Renzi, E., Gentilini, M., Faglioni, P., \& Barbieri, C. (1989). Attentional shift towards the rightmost stimuli in patients with left visual neglect. Cortex, 25, 231-237.

Dobler, V.B., Anker, S., Gilmore, J., Robertson, I.H., Atkinson, J., \& Manly, T. (2005). Asymmetric deterioration of spatial awareness with diminishing levels of alertness in normal children and children with ADHD. Journal of Child Psychology and Psychiatry, 46, 1230-1248.

Dobler, V.B., Manly, T., Verity, C., Woolrych, J., \& Robertson, I.H. (2003). Modulation of spatial attention in a child with developmental unilateral neglect. Developmental Medicine and Child Neurology, 45, 282-288. 
Fernandez-Duque, D. \& Posner, M.I. (2001). Brain imaging of attentional networks in normal and pathological states. Journal of Clinical and Experimental Neuropsychology, 23, 74-93.

Fimm, B., Willmes, K., \& Spijkers, W. (2006). The effect of low arousal on visuo-spatial attention. Neuropsychologia, 44 , 1261-1268.

Friedrich, F.J., Egly, R., Rafal, R.D., \& Beck, D. (1998). Spatial attention deficits in humans: A comparison of superior parietal and temporal-parietal junction lesions. Neuropsychology, 12, 193-207.

Gitelman, D.R., Nobre, A.C., Parrish, T.B., LaBar, K.S., Kim, Y.H., Meyer, J.R., \& Mesulam, M. (1999). A large-scale distributed network for covert spatial attention: Further anatomical delineation based on stringent behavioural and cognitive controls. Brain, 122, 1093-1106.

Gitelman, D.R., Parrish, T.B., Friston, K.J., \& Mesulam, M.M. (2002). Functional anatomy of visual search: Regional segregations within the frontal eye fields and effective connectivity of the superior colliculus. Neuroimage, 15, 970-982.

Goodale, M.A. \& Milner, A.D. (1992). Separate visual pathways for perception and action. Trends in Neurosciences, 15, 20-25.

Halligan, P.W. \& Marshall, J.C. (1991). Left neglect for near but not far space in man. Nature, 350, 498-500.

Heber, I.A., Wolter, M., Kuhlen, T., \& Fimm, B. (2007). Asymmetries of covert attention in virtual space. Annual Review of CyberTherapy and Telemedicine, 5, 343.

Heller, O. (1985). Hörfeldaudiometrie mit dem Verfahren der Kategorienunterteilung. Psychologische Beiträge, 27, 478-493.

Karnath, H.O., Niemeier, M., \& Dichgans, J. (1998). Space exploration in neglect. Brain, 121, 2357-2367.

Kirk, R.E. (1994). Experimental Design (3rd ed.), Procedures for the Behavioral Sciences. Pacific Grove, CA: Brooks/Cole.

Ladavas, E. \& Farne, A. (2004). Visuo-tactile representation of near-the-body space. Journal of Physiology, Paris, 98, 161-170.

Longo, M.R. \& Lourenco, S.F. (2006). On the nature of near space: Effects of tool use and the transition to far space. Neuropsychologia, 44, 977-981.

Losier, B.J. \& Klein, R.M. (2004). Covert orienting within peripersonal and extrapersonal space: Young adults. Brain Research. Cognitive Brain Research, 19, 269-274.

Manly, T., Dobler, V.B., Dodds, C.M., \& George, M.A. (2005). Rightward shift in spatial awareness with declining alertness. Neuropsychologia, 43, 1721-1728.

Maringelli, F., McCarthy, J., Steed, A., Slater, M., \& Umilta, C. (2001). Shifting visuo-spatial attention in a virtual threedimensional space. Brain Research. Cognitive Brain Research, 10, 317-322.

Marrocco, R.T., Witte, E.A., \& Davidson, M.C. (1994). Arousal systems. Current Opinion in Neurobiology, 4, 166-170.

Mennemeier, M., Wertman, E., \& Heilman, K.M. (1992). Neglect of near peripersonal space. Evidence for multidirectional attentional systems in humans. Brain, 115, 37-50.

Morrison, J.H. \& Foote, S.L. (1986). Noradrenergic and serotoninergic innervation of cortical, thalamic, and tectal visual structures in Old and New World monkeys. The Journal of Comparative Neurology, 243, 117-138.

Myors, B. (1999). Timing accuracy of PC programs running under DOS and Windows. Behavior Research Methods, Instruments, \& Computers, 31, 322-328.

Nigg, J.T., Swanson, J.M., \& Hinshaw, S.P. (1997). Covert visual spatial attention in boys with attention deficit hyperactivity disorder: Lateral effects, methylphenidate response and results for parents. Neuropsychologia, 35, 165-176.

Oldfield, R.C. (1971). The assessment and analysis of handedness: The Edinburgh inventory. Neuropsychologia, 9, 97-113.

Petersen, S.E., Robinson, D.L., \& Currie, J.N. (1989). Influences of lesions of parietal cortex on visual spatial attention. Experimental Brain Research, 76, 267-280.

Posner, M.I. (1980). Orienting of attention. Quarterly Journal of Experimental Psychology, 32, 3-25.

Posner, M.I. \& Petersen, S.E. (1990). The attention system of the human brain. Annual Review of Neuroscience, 13, 25-42.

Posner, M.I., Walker, J.A., Friedrich, F.J., \& Rafal, R.D. (1984). Effects of parietal injury on covert orienting of attention. The Journal of Neuroscience, 4, 1863-1874.

Previc, F.H. (1998). The neuropsychology of 3-D space. Psychological Bulletin, 124, 123-164.

Quinlan, D.J. \& Culham, J.C. (2007). fMRI reveals a preference for near viewing in the human parieto-occipital cortex. Neuroimage, 36, 167-187.

Robertson, I.H., Mattingley, J.B., Rorden, C.H., \& Driver, J. (1998). Phasic alerting of neglect patients overcomes their spatial deficit in visual awareness. Nature, 395, 169-172.

Shelton, P.A., Bowers, D., \& Heilman, K.M. (1990). Peripersonal and vertical neglect. Brain, 113, 191-205.

Sheppard, D.M., Bradshaw, J.L., Mattingley, J.B., \& Lee, P. (1999). Effects of stimulant medication on the lateralisation of line bisection judgements of children with attention deficit hyperactivity disorder. Journal of Neurology, Neurosurgery, \& Psychiatry, 66, 57-63.

Sturm, W., de Simone, A., Krause, B.J., Specht, K., Hesselmann, V., Radermacher, I., Herzog, H., Tellmann, L., MullerGartner, H.W., \& Willmes, K. (1999). Functional anatomy of intrinsic alertness: Evidence for a fronto-parietal-thalamicbrainstem network in the right hemisphere. Neuropsychologia, 37, 797-805.

Sturm, W. \& Willmes, K. (2001). On the functional neuroanatomy of intrinsic and phasic alertness. Neuroimage, 14, 76-84.

Thiebaut de Schotten, M., Urbanski, M., Duffau, H., Volle, E., Levy, R., Dubois, B., \& Bartolomeo, P. (2005). Direct evidence for a parietal-frontal pathway subserving spatial awareness in humans. Science, 309, 2226-2228.

Thiel, C.M., Zilles, K., \& Fink, G.R. (2004). Cerebral correlates of alerting, orienting and reorienting of visuospatial attention: An event-related fMRI study. Neuroimage, 21, 318-328.

Thimm, M., Fink, G.R., Kust, J., Karbe, H., \& Sturm, W. (2006). Impact of alertness training on spatial neglect: A behavioural and fMRI study. Neuropsychologia, 44, 1230-1246.

Thomas, M., Sing, H., Belenky, G., Holcomb, H., Mayberg, H., Dannals, R., Wagner, H., Thorne, D., Popp, K., Rowland, L., Welsh, A., Balwinski, S., \& Redmond, D. (2000). Neural basis of alertness and cognitive performance impairments during sleepiness. I. Effects of $24 \mathrm{~h}$ of sleep deprivation on waking human regional brain activity. Journal of Sleep Research, 9, 335-352.

Vallar, G. (2001). Extrapersonal visual unilateral spatial neglect and its neuroanatomy. Neuroimage, 14, 52-58.

Vallar, G., Bottini, G., \& Paulesu, E. (2003). Neglect syndromes: The role of the parietal cortex. Advances in Neurology, 93, 293-319.

Valvoda, J.T., Assenmacher, I., Dohle, C., Kuhlen, T., \& Bischof, C.H. (2003). NeuroVRAC-A comprehensive approach to virtual reality-based neurological assessment and treatment systems. Studies in Health Technology \& Informatics, 94, 370-372. 
Valvoda, J.T., Assenmacher, I., Kuhlen, T., \& Bischof, C.H. (2004). Reaction-time measurement and real-time data acquisition for neuroscientific experiments in virtual environments. Studies in Health Technology \& Informatics, 98, 391-393.

Van Someren, E.J. (2000). More than a marker: Interaction between the circadian regulation of temperature and sleep, age-related changes, and treatment possibilities. Chronobiology International, 17, 313-354.

Voeller, K.K. \& Heilman, K.M. (1988). Attention deficit disorder in children: A neglect syndrome? Neurology, 38, 806-808.

von Zerssen, D. (1976). Die Befindlichkeitsskala. Weinheim, Germay: Beltz.

Vuilleumier, P., Valenza, N., Mayer, E., Reverdin, A., \& Landis, T. (1998). Near and far visual space in unilateral neglect. Annals of Neurology, 43, 406-410.
Weiss, P.H., Marshall, J.C., Wunderlich, G., Tellmann, L., Halligan, P.W., Freund H.J., Zilles K., \& Fink, G.R. (2000). Neural consequences of acting in near versus far space: A physiological basis for clinical dissociations. Brain, 123, 2531-2541.

Wolter, M., Armbruester, C., Valvoda, J.T., \& Kuhlen, T. (2007). High Ecological Validity and Accurate Stimulus Control in VR-based Psychological Experiments. Proceedings of Eurographics Symposium on Virtual Environments, Immersive Projection Technology Workshop (EGVE'07). Elmsford, NY: Pergamon Press.

Zimmermann, P. \& Fimm, B. (2002). Test for Attentional Performance (TAP). Version 1.7. Herzogenrath, Germany: Psytest. 